\title{
Adaptive Unipolar MIMO-OFDM for Visible Light Communications
}

\author{
Mohamed Al-Nahhal ${ }^{1}$, Ertugrul Basar ${ }^{2}$, and Murat Uysal ${ }^{1}$ \\ ${ }^{1}$ Department of Electrical and Electronics Engineering, Ozyegin University, Istanbul, Turkey, 34794 \\ ${ }^{2}$ CoreLab, Department of Electrical and Electronics Engineering, Koç University, Istanbul, Turkey, 34450 \\ (e-mails: mohamed.al-nahhal@ozu.edu.tr, ebasar@ku.edu.tr, murat.uysal@ozyegin.edu.tr)
}

\begin{abstract}
Unipolar orthogonal frequency division multiplexing (U-OFDM) appears as an attractive optical OFDM solution for emerging visible light communication (VLC) systems. This paper proposes spectral efficiency improvement for U-OFDM systems by applying adaptive transmission over realistic VLC links. This adaptive transmission includes switching among a number of multiple-input multiple-output (MIMO) modes combined with appropriate modulation size selection. The considered MIMO modes are repetition coding, spatial modulation, and spatial multiplexing, where each mode supports different modulation sizes. The selection of the corresponding MIMO mode and its modulation size is based on the received signal-to-noise ratio and target bit error rate. The proposed U-OFDM system is applied over different VLC MIMO setups with realistic channel models for $8 \times 8,4 \times 4$ and $2 \times 2$ MIMO systems. Our simulation results show that the proposed adaptive system provides a significant spectral efficiency improvement over stand-alone $\mathrm{U}$ OFDM MIMO modes/setups.
\end{abstract}

Index Terms-Visible light communication, unipolar OFDM, adaptive transmission, MIMO modes switching, repetition coding, spatial modulation, spatial multiplexing.

\section{INTRODUCTION}

$\mathbf{V}$ ISIBLE light communications (VLC) is a promising solution for wireless communication systems in beyond 5G networks, and provides high energy efficiency, low implementation cost, and high-speed data transmission [1]. VLC systems can be integrated into the existing radio frequency (RF) based wireless communication systems in order to satisfy the increasing high capacity demands. In VLC systems, light emitting diodes (LEDs) and photodetectors (PDs) are used as transmitters and receivers, respectively. This means that the LEDs are used for both illumination and communication purposes. Due to the characteristics of LEDs, only unipolar signals can be conveyed through LEDs. Consequently, VLC systems rely on intensity modulation and direct detection for transmission [2].

Initial studies on VLC systems focused on very simple modulation formats, such as on-off keying, to deal with the constraints of LEDs [1]. However, the nature of VLC channels is frequency-selective, which causes significant degradation in the VLC system performance due to critical inter-symbolinterference (ISI). To mitigate the ISI of the VLC system, several schemes based on pulse modulation have been proposed [3]. Orthogonal frequency-division multiplexing (OFDM) has been considered in [4] to mitigate the ISI of the VLC system. In [5], the concept of multiple-input multiple-output (MIMO) transmission has been presented in order to achieve high data rate for indoor environments.

In future $5 \mathrm{G}$ communication networks and beyond, spectral efficiency needs further improvement [6]. One of the techniques that improves the spectral efficiency of VLC systems is the adaptive transmission technique [7]-[11]. Adaptive transmission utilizes one or more transmission parameters such as transmit power, modulation size, etc. in order to improve the spectral efficiency based on the channel quality. In [7], adaptive transmission has been applied to the singlecarrier spatial multiplexing (SMUX) MIMO system over the VLC link, while [8] proposed a spatial modulation (SM) MIMO system based on adaptive transmission. An energy efficiency improvement method for the MIMO VLC system has been presented in [9] based on adaptive transmission. In [10] and [11], adaptive transmission has been proposed for MIMO DC-biased optical OFDM (DCO-OFDM) VLC system. The proposed adaptive system in [10] considered the SMUX MIMO mode to improve the system performance based on bit and power loading. Whereas in [11], the proposed adaptive system supported both repetition coding (RC) and SMUX MIMO modes for spectral efficiency enhancement.

The unipolar OFDM (U-OFDM) scheme is more power efficient than DCO-OFDM since it does not require a DC bias as DCO-OFDM [2, Ch. 12]. As a result, U-OFDM can achieve a lower bit error rate (BER) than DCO-OFDM.

Against this background, this paper proposes an adaptive transmission technique for MIMO VLC system in conjunction with U-OFDM to exploit the U-OFDM benefits. The proposed adaptive MIMO U-OFDM VLC system is implemented to support three different MIMO modes (RC, SMUX, and SM) that enable a set of different modulation sizes. Depending on the received signal-to-noise ratio (SNR) and target BER, the proposed adaptive transmission system switches between the available MIMO modes and adjusts its modulation size to achieve higher spectral efficiency.

The structure of this paper ${ }^{1}$ is as follows: Section II presents the proposed adaptive MIMO U-OFDM VLC system. Section III describes the indoor MIMO VLC channel model. In Section IV, our numerical results are given. Finally, the conclusion of this work is presented in Section V.

\footnotetext{
${ }^{1}$ This work was supported by the Turkish Scientific and Research Council (TUBITAK) under grant $215 \mathrm{E} 311$.
} 


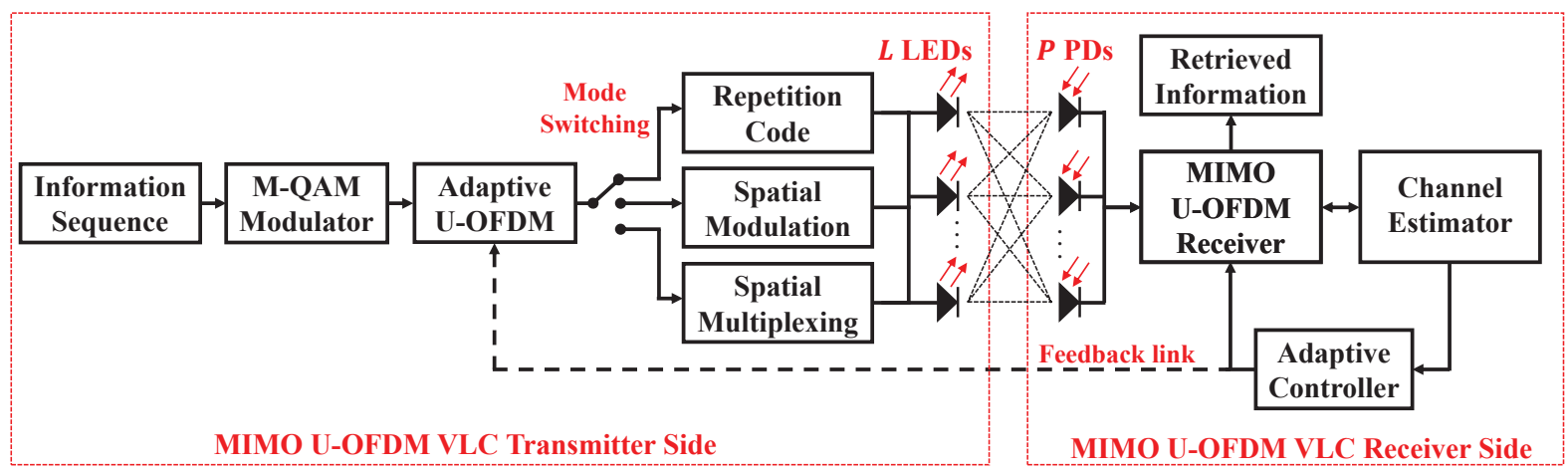

Fig. 1: Block diagram of the proposed adaptive MIMO U-OFDM VLC system.

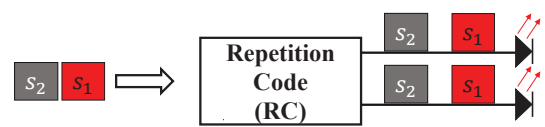

(a)

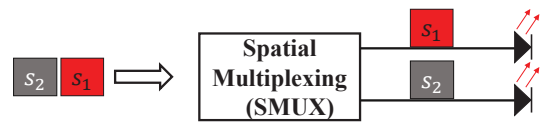

(b)



(c)

Fig. 2: (a) RC, (b) SMUX and (c) SM.

\section{Proposed System Model}

Consider a MIMO system with $L$ LEDs at the transmitter and $P$ PDs at the receiver as shown in Fig. 1. The proposed adaptive MIMO system is designed to support three different MIMO modes (i.e., RC, SM, and SMUX). The RC mode is the simplest MIMO mode [5], in which all LEDs simultaneously emit the same signal for achieving diversity gain through repetition (see Fig. 2a). In the SMUX mode [12, Ch. 10], independent information signals are transmitted simultaneously from all LEDs to achieve multiplexing gain (see Fig. 2b). For the SM mode [13], the transmitter chooses randomly only one of the LEDs as active and the index of the activated LED carries information in addition to the modulated signal transmitted from the selected LED (see Fig. 2c). The proposed adaptive system selects the appropriate MIMO mode and modulation size based on the received SNR, then, reports this information to the transmitter through a feedback link.

\section{A. MIMO U-OFDM Transmitter/Receiver}

The adaptive system architecture is built on the U-OFDM system. In the U-OFDM system, binary information is first mapped to complex symbols using $M$-ary quadrature amplitude modulation ( $M$-QAM) with the modulation size $M$ and the average electrical symbol energy $E$. The complex-valued modulated symbols are applied to Hermitian symmetry before inverse fast Fourier transform (IFFT), for extracting a realvalued bipolar signal. A cyclic prefix with the size of $N_{\mathrm{CP}}$ is appended to real-valued bipolar signal in order to overcome the ISI. The resulting real-valued bipolar signal is converted into a unipolar signal by doubling the original OFDM frame. The first OFDM frame refers to the positive samples and the second one represents negative samples. In the first frame, the negative samples set as inactive (i.e., set to zero). In the second frame, the positive samples set as inactive and the negative samples are multiplied by -1 to be positive. Then, the resulting signal propagates through the optical VLC channel to the PDs. At the receiver side, the negative frame of U-OFDM signal is subtracted from the positive frame. After removing the cyclic prefix, the FFT output at $p$-th PD, $p=1,2, \ldots, P$ is given by

$$
y_{p}(n)=\sqrt{\frac{E}{L_{a}}} \sum_{l} h_{p, l}(n) s_{l}(n)+w_{p}(n), n=1,2, \ldots, N
$$

where $y_{p}(n)$ is the received signal for the $n$-th subcarrier at the $p$-th PD and the total number of subcarriers (FFT size) is denoted by $N$. In (1), $s_{l}(n)$ represents the transmitted signal from the $l$-th LED for the $n$-th subcarrier and drawn from $M$-QAM constellation. In case of RC and SMUX modes, $l=1,2, \ldots, L$, where $s_{1}(n)=s_{2}(n)=\cdots=s_{L}(n)$ for RC and $s_{1}(n), s_{2}(n), \cdots, s_{L}(n)$ are independent symbols for SMUX. Whereas in case of the SM mode, $l \in\{1,2, \ldots, L\}$ corresponds to the activated LED index. In (1), $L_{a}$ is the activated LEDs, where $L_{a}=L$ in case of the RC and SMUX modes, while $L_{a}=1$ for SM mode. Here, $h_{p, l}(n)$ represents the $n$-th subcarrier VLC channel gain between the $p$-th PD and the $l$-th LED, $w_{p}(n) \sim \mathcal{N}\left(0, N_{0}\right)$ is the additive white Gaussian noise term at the $p$-th PD with zero mean and variance $N_{0}$, and $N_{0}$ denotes the noise power spectral density.

At the receiver side, the maximum likelihood (ML) detector is used for RC, SMUX, and SM. The ML decision for RC and SMUX is given by

$$
\hat{\mathbf{s}}(n)^{(\mathrm{ML})}=\underset{\mathbf{s}(n)}{\arg \min }\|\mathbf{y}(n)-\mathbf{H}(n) \mathbf{s}(n)\|^{2},
$$

where $\mathbf{y}(n)$ is the received U-OFDM signal vector with dimensions $P \times 1$, and each element of this vector is given by $y_{p}(n)$ that is defined earlier in $(1), \mathbf{s}(n)=$ 
$\left[\begin{array}{llll}s_{1}(n) & s_{2}(n) & \ldots & s_{L}(n)\end{array}\right]^{\mathrm{T}}$ is the transmitted $\mathrm{U}$ OFDM signal vector with dimensions $L \times 1$, and $[\cdot]^{\mathrm{T}}$ denotes the transpose. Here, $\mathbf{H}(n)$ denotes the MIMO channel gains matrix with dimensions $P \times L$, where each matrix entry is defined by $h_{p, l}(n)$. For the SM mode, the ML decision should be modified where the search is over both LED index and modulated signal as

$$
\left[\hat{l}^{(\mathrm{ML})}, \hat{s}(n)^{(\mathrm{ML})}\right]=\underset{\substack{s(n) \\ l \in\{1,2, \ldots L\}}}{\arg \min }\left\|\mathbf{y}(n)-\mathbf{h}_{l}(n) s(n)\right\|^{2} .
$$

Here, $\mathbf{h}_{l}(n)$ is the $l$-th column of $\mathbf{H}(n)$. The ML detector of SMUX might be prohibitive for higher modulation sizes or larger MIMO setups due to its high complexity [14]. As a result, the zero-forcing (ZF) equalizer is used for an alternative with lower detection cost. The ZF decision is expressed as

$$
\hat{\mathbf{s}}(n)^{(\mathrm{ZF})}=\left((\mathbf{H}(n))^{\mathrm{T}} \mathbf{H}(n)\right)^{-1}\left((\mathbf{H}(n))^{\mathrm{T}} \mathbf{y}(n)\right) .
$$

For further performance improvement, the optimal ordering successive interference cancellation (OSIC) is adopted after $\mathrm{ZF}$ equalization as described in [12, Sec. (11.2)].

\section{B. Adaptive Transmission Technique}

In this subsection, the proposed adaptive transmission technique for MIMO U-OFDM VLC system is described. The basic idea of adaptive transmission is to switch the transmission parameters, such as MIMO modes or/and MIMO setups, based on the received SNR and a target BER, $P_{\text {th }}$. A feedback link is required for transferring the necessary information to the transmitter. VLC system usually employs the RF, or infrared waves as a feedback link [15]. Thus, the feedback link does not provide additional overhead to the VLC system. The steps of the adaptive transmission technique are described as follows:

Step 1: Define threshould BER value $P_{\text {th }}$ for given $E$ and $\mathbf{H}$. Here, $\mathbf{H}=\mathbf{H}(n) \in \mathbb{R}^{P \times L}$ for $\forall n$ and $\mathrm{E}\left[\left\|\mathbf{h}_{l}\right\|^{2}\right]$ is constant, where $l=1,2, \ldots, L$ and $\mathrm{E}[\cdot]$ denotes the expectation operator.

Step 2: Calculate the received SNR, $\gamma$, as

$$
\gamma=\frac{E}{L N_{0}} \sum_{p=1}^{P}\left|\sum_{l=1}^{L} h_{p, l}\right|^{2} .
$$

The (5) is valid for all MIMO modes, $\Xi \in\{$ RC,SM,SMUX $\}$, based on $\mathbf{H}$ that will be defined in Section III.

Step 3: Find the maximum constellation size $M_{\Xi}^{(\max )}$, which satisfies $P_{\text {th }}$. Then, calculate the corresponding spectral efficiency for each MIMO mode, $\eta_{\Xi}$, in bits $/ \mathrm{sec} / \mathrm{Hz}(\mathrm{b} / \mathrm{s} / \mathrm{Hz})$ as [2, Ch. 12]

$$
\begin{gathered}
\eta_{\mathrm{RC}}=\frac{1}{2}\left(\frac{N / 2-1}{N+N_{\mathrm{CP}}}\right) \log _{2}\left(M_{\mathrm{RC}}^{(\max )}\right), \\
\eta_{\mathrm{SM}}=\frac{1}{2}\left(\frac{N / 2-1}{N+N_{\mathrm{CP}}}\right) \log _{2}\left(N_{t} M_{\mathrm{SM}}^{(\max )}\right), \\
\eta_{\mathrm{SMUX}}=\frac{N_{t}}{2}\left(\frac{N / 2-1}{N+N_{\mathrm{CP}}}\right) \log _{2}\left(M_{\mathrm{SMUX}}^{(\max )}\right) .
\end{gathered}
$$

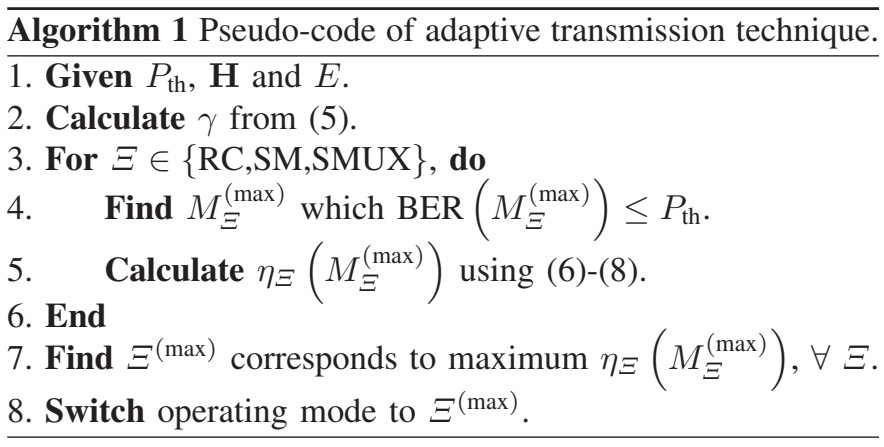

Step 4: Find the maximum spectral efficiency resulted from (6)-(8) and determine its mode, $\Xi^{(\max )}$. Then, the adaptive transmission technique switches the operation mode to $\Xi^{(\max )}$.

Our adaptive transmission technique is summarized in Algorithm 1.

\section{INDOOR MIMO VLC CHANNEL}

In this paper, the MIMO setups of $8 \times 8,4 \times 4$ and $2 \times 2$ are considered. The indoor VLC channels developed in [11] are used for our simulations. The considered MIMO setups are chosen from subsets of the general $16 \times 16$ MIMO system in [11]. The normalized channel matrices (i.e., $\mathrm{E}\left[\left\|\mathbf{h}_{l}\right\|^{2}\right]$ is fixed for all $l=1,2, \ldots, L)$ for $8 \times 8,4 \times 4$ and $2 \times 2 \mathrm{MIMO}$ setups are respectively given by

$\mathbf{H}=10^{-6} \times$

$\left(\begin{array}{llllllll}0.377 & 0.483 & 0.446 & 0.523 & 0.400 & 0.466 & 0.273 & 0.430 \\ 0.455 & 0.407 & 0.395 & 0.427 & 0.384 & 0.362 & 0.304 & 0.141 \\ 0.236 & 0.243 & 0.300 & 0.136 & 0.356 & 0.384 & 0.388 & 0.382 \\ 0.316 & 0.202 & 0.300 & 0.119 & 0.353 & 0.164 & 0.349 & 0.478 \\ 0.211 & 0.206 & 0.270 & 0.266 & 0.327 & 0.362 & 0.428 & 0.426 \\ 0.240 & 0.224 & 0.285 & 0.343 & 0.304 & 0.338 & 0.426 & 0.450 \\ 0.391 & 0.501 & 0.388 & 0.413 & 0.345 & 0.344 & 0.312 & 0.122 \\ 0.490 & 0.397 & 0.401 & 0.389 & 0.350 & 0.338 & 0.315 & 0.153\end{array}\right)$,

$$
\begin{gathered}
\mathbf{H}=10^{-6} \times\left(\begin{array}{cccc}
0.5356 & 0.6831 & 0.3838 & 0.5762 \\
0.3354 & 0.3440 & 0.5461 & 0.5121 \\
0.3414 & 0.3161 & 0.5987 & 0.6031 \\
0.6958 & 0.5614 & 0.4428 & 0.2052
\end{array}\right), \\
\mathbf{H}=10^{-6} \times\left(\begin{array}{cc}
0.6958 & 0.7644 \\
0.7182 & 0.6447
\end{array}\right) .
\end{gathered}
$$

\section{NumericAl RESUlTS AND Discussions}

In this section, the spectral efficiency and date rate of the proposed adaptive U-OFDM MIMO VLC system are evaluated for different MIMO setups mentioned in Section III. The data rate equals $\eta \times B(\mathrm{~b} / \mathrm{s})$, where $B$ is the system bandwidth. In Figs. 4 - 7, the left and right vertical axis represent the spectral efficiency and data rate, respectively; while the horizontal axis represents $\gamma$ given from (5). The channel state information is assumed to be perfectly known at the receiver side. The ML detector is used for simulating the BER performances of $\mathrm{RC}$ and SM MIMO modes for all modulation sizes. Whereas 


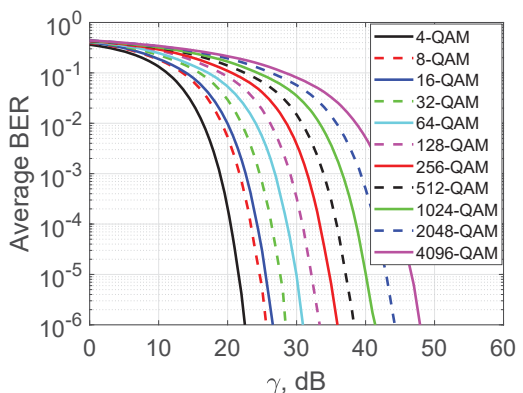

(a)

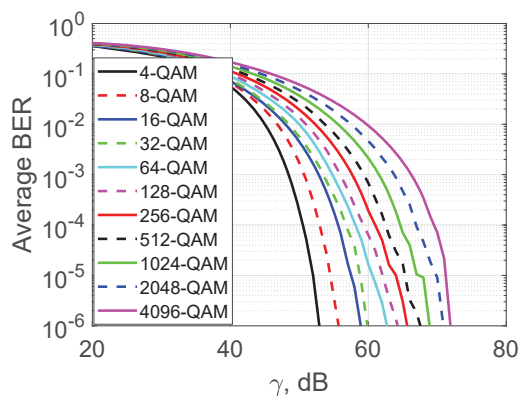

(b)

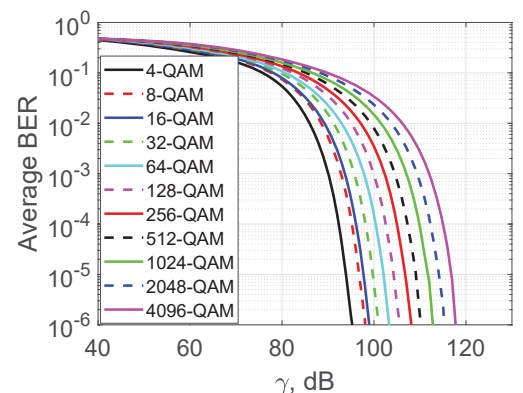

(c)

Fig. 3: BERs performances for U-OFDM VLC system of $8 \times 8$ MIMO modes (a) RC, (b) SM, and (c) SMUX.

TABLE I: Simulation parameters.

\begin{tabular}{lcc}
\hline Parameters & Symbol & Value \\
\hline \hline FFT size & $N$ & $1024[4]$ \\
\hline Cyclic prefix size & $N_{\mathrm{CP}}$ & $4[4]$ \\
\hline Target BER & $P_{\mathrm{th}}$ & $10^{-6}$ \\
\hline System bandwidth & $B$ & $20 \mathrm{MHz}[4]$ \\
\hline Available modulation sizes & $M$ & 4 to 4096 \\
\hline MIMO setup & $N_{r} \times N_{t}$ & $2 \times 2,4 \times 4$ and $8 \times 8$ \\
\hline
\end{tabular}

the simulation of BER performance for the SMUX MIMO mode utilizes three different detectors (ML, ZF, and ZF-OSIC) according to the complexity. For fair comparison, we consider fixed transmit power for all modes. The simulation parameters are provided in Table I.

Fig. 3 depicts the BER performance for different modes of the $8 \times 8$ MIMO-VLC system utilizing U-OFDM. The proposed adaptive transmission technique uses these BER results to find $M_{\Xi}^{(\max )}$ that satisfies $P_{\text {th }}$ for $\forall \Xi$. For instance, at $\gamma=60 \mathrm{~dB}$ and $P_{\text {th }}=10^{-6}$, the maximum constellation size for the RC mode is 4096 and provides $2.9825 \mathrm{~b} / \mathrm{s} / \mathrm{Hz}$ (i.e., $M_{\mathrm{RC}}^{(\max )}=4096$ and $\eta_{\mathrm{RC}}=2.9825 \mathrm{~b} / \mathrm{s} / \mathrm{Hz}$ ). Whereas for $\mathrm{SM}$ the mode, $M_{\mathrm{SM}}^{(\max )}=32$ and $\eta_{\mathrm{SM}}=1.9883 \mathrm{~b} / \mathrm{s} / \mathrm{Hz}$. In the SMUX mode, there is no $M_{\mathrm{SMUX}}^{(\max )}$ that satisfies $P_{\mathrm{th}}$. Therefore, at $\gamma=60 \mathrm{~dB}$, the proposed algorithm selects the $\mathrm{RC}$ mode as the operation mode. It should be noted that in a real system, the proposed algorithm switches between the modes/setups and uses the theoretical equations instead of the BER curves.

In Fig. 4, the spectral efficiency and the corresponding data rate of the proposed adaptive transmission system (indicated by dotted line) are depicted for the $8 \times 8$ MIMO U-OFDM VLC system. For this setup, the $\mathrm{ZF}$ equalizer is employed for the SMUX in all modulation sizes. As benchmarks, the stand-alone RC, SM and SMUX systems are considered. It is observed from Fig. 4 that in the low SNR region, RC outperforms both SM and SMUX as a result of diversity gains. Specifically, the adaptive system selects the RC mode when $\gamma<68 \mathrm{~dB}$. In the ranges of $68 \mathrm{~dB}$ to $95 \mathrm{~dB}$, the adaptive system selects the SM mode. After $95 \mathrm{~dB}$, the SMUX significantly outperforms its counterparts by taking advantage

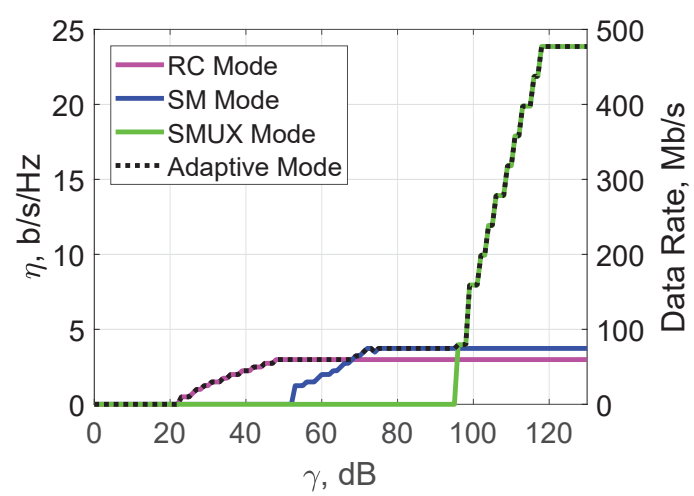

Fig. 4: Spectral efficiency and date rate for $8 \times 8$ MIMO UOFDM VLC system.

of multiplexing gains and is therefore selected as the operation mode. It should be noted that the spectral efficiencies of the RC and SM saturate respectively at $48 \mathrm{~dB}$ and $75 \mathrm{~dB}$, where 4096-QAM is employed. The maximum achievable spectral efficiency for the RC and SM are respectively 2.982 and $3.728 \mathrm{~b} / \mathrm{s} / \mathrm{Hz}$ which respectively correspond to data rates of 59.64 and $74.56 \mathrm{Mb} / \mathrm{s}$. The SMUX saturates at $118 \mathrm{~dB}$ and the achievable maximum spectral efficiency of $23.86 \mathrm{~b} / \mathrm{s} / \mathrm{Hz}$ corresponds to a data rate of $477.2 \mathrm{Mb} / \mathrm{s}$.

Figs. 5 and 6 show the spectral efficiency and the corresponding data rate of $4 \times 4$ and $2 \times 2$ MIMO U-OFDM VLC systems, respectively. The SMUX uses the ML detector for low modulation sizes (below $M=128$ and 16 in $2 \times 2$ and $4 \times 4$ MIMO setups, respectively) and ZF-OSIC detector for the high modulation sizes. In Fig. 5, the proposed adaptive transmission system selects the RC mode for $\gamma<64 \mathrm{~dB}$ as the operation mode. The SM mode is chosen in the range of $64 \mathrm{~dB}$ to $73 \mathrm{~dB}$. For $\gamma>73 \mathrm{~dB}$, the adaptive transmission system selects the SMUX mode. The maximum achievable spectral efficiency and corresponding data rate for the $4 \times 4$ system in Fig. 5 decrease to half in comparison with the $8 \times 8$ system in Fig. 4. In Fig. 6, the adaptive transmission system chooses the RC mode for $\gamma<54 \mathrm{~dB}$. The proposed adaptive transmission system chooses the SMUX mode when $\gamma>54$ dB. In the $2 \times 2$ system (Fig. 6), the maximum achievable spectral efficiency reduces from 23.86 to $5.965 \mathrm{~b} / \mathrm{s} / \mathrm{Hz}$ and 


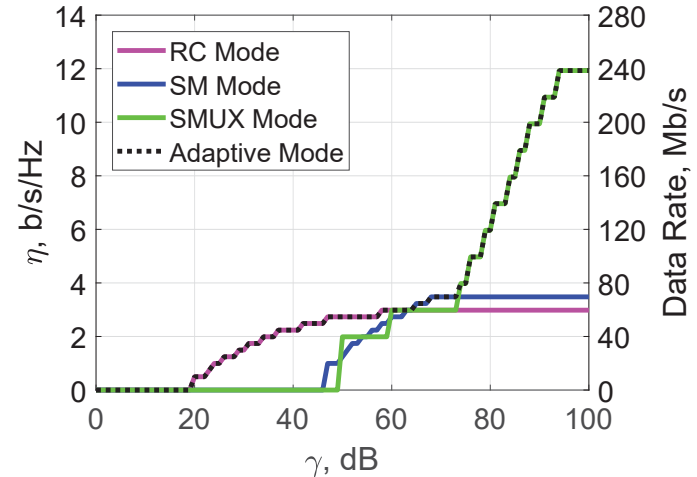

Fig. 5: Spectral efficiency and date rate for $4 \times 4$ MIMO UOFDM VLC system.

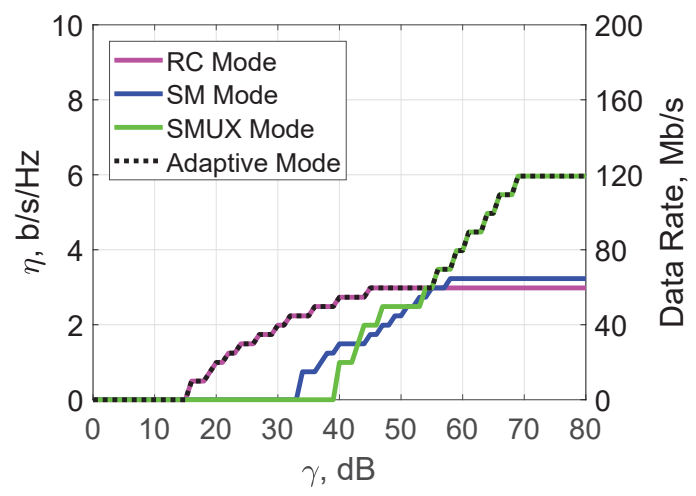

Fig. 6: Spectral efficiency and date rate for $2 \times 2 \mathrm{MIMO}$ UOFDM VLC system.

corresponding data rate decreases from 477.2 to $119.3 \mathrm{Mb} / \mathrm{s}$, compared to $8 \times 8 \mathrm{MIMO}$ system.

For further spectral efficiency improvement, switching between the MIMO system setups of $2 \times 2,4 \times 4$ and $8 \times 8$ is investigated in Fig. 7. The proposed adaptive transmission system selects the $2 \times 2$ MIMO setup when $\gamma<79 \mathrm{~dB}$ which achieves $5.965 \mathrm{~b} / \mathrm{s} / \mathrm{Hz}$ with a data rate of $119.3 \mathrm{Mb} / \mathrm{s}$. When $\gamma$ lies between $79 \mathrm{~dB}$ and $104 \mathrm{~dB}$, the adaptive transmission system chooses the $4 \times 4$ MIMO setup and the achievable

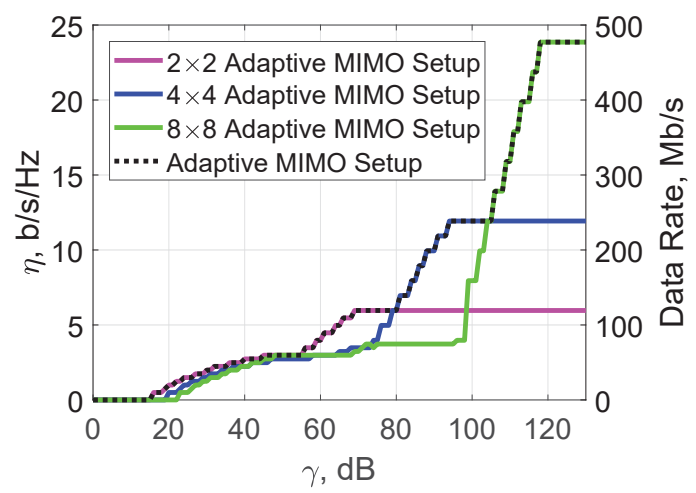

Fig. 7: Spectral efficiency and date rate of the adaptive setup technique for MIMO U-OFDM VLC system. spectral efficiency is $11.93 \mathrm{~b} / \mathrm{s} / \mathrm{Hz}$ with a data rate of 238.6 $\mathrm{Mb} / \mathrm{s}$. Finally, the proposed adaptive transmission system activated the $8 \times 8$ MIMO setup at $\gamma>104 \mathrm{~dB}$, which provides the maximum spectral efficiency and data rate as $23.86 \mathrm{~b} / \mathrm{s} / \mathrm{Hz}$ and $477.2 \mathrm{Mb} / \mathrm{s}$, respectively.

It can be observed from these figures that the proposed adaptive transmission system benefits from RC, SM, and SMUX by employing mode switching relying on the received SNR. Consequently, the proposed adaptive MIMO U-OFDM VLC system exhibits a superior spectral efficiency over the stand-alone modes/setups.

\section{CONCLUSION}

This paper has proposed an adaptive transmission mechanism for the MIMO U-OFDM VLC system over different realistic VLC channel setups. The proposed adaptive transmission system has been designed to maximize the spectral efficiency of the system. The obtained spectral efficiency improvement is a result of switching between RC, SM and SMUX MIMO modes by satisfying the target BER based on the received SNR. Moreover, switching between different MIMO system setups has been considered for further spectral efficiency improvement. Our simulation results have demonstrated that the proposed adaptive system provides a significant spectral efficiency improvement compared to stand-alone modes/setups.

\section{REFERENCES}

[1] T. Komine and M. Nakagawa, "Fundamental analysis for visible-light communication system using LED lights," IEEE Trans. Consum. Electron., vol. 50, no. 1, pp. 100-107, Feb. 2004.

[2] M. Uysal et al., Optical Wireless Communications: An Emerging Technology. Springer, 2016.

[3] S. Rajagopal, R. D. Roberts, and S.-K. Lim, "IEEE 802.15.7 visible light communication: Modulation schemes and dimming support," IEEE Commun. Mag., vol. 50, no. 3, pp. 72-82, Mar. 2012.

[4] R. Mesleh, H. Elgala, and H. Haas, "On the performance of different OFDM based optical wireless communication systems," IEEE/OSA J. Opt. Commun. Netw., vol. 3, no. 8, pp. 620-628, Aug. 2011.

[5] T. Fath and H. Haas, "Performance comparison of MIMO techniques for optical wireless communications in indoor environments," IEEE Trans. Commun., vol. 61, no. 2, pp. 733-742, Feb. 2013.

[6] S. Al-Ahmadi, O. Maraqa, M. Uysal, and S. M. Sait, "Multi-user visible light communications: State-of-the-art and future directions," IEEE Access, vol. 6, pp. 70555-70571, Nov. 2018.

[7] K.-H. Park et al., "On the power and offset allocation for rate adaptation of spatial multiplexing in optical wireless MIMO channels," IEEE Trans. Commun., vol. 61, no. 4, pp. 1535-1543, Apr. 2013.

[8] P. F. Mmbaga, J. Thompson, and H. Haas, "Performance analysis of indoor diffuse VLC MIMO channels using angular diversity detectors," J. Lightw. Technol., vol. 34, no. 4, pp. 1254-1266, Feb. 2016.

[9] I. Tavakkolnia, C. Chen, R. Bian, and H. Haas, "Energy-efficient adaptive MIMO-VLC technique for indoor LiFi applications," 25th Int. Conf. Telecommun. (ICT), St. Malo, France, Jun. 2018, pp. 331-335.

[10] Y. Hong, T. Wu, and L.-K. Chen, "On the performance of adaptive MIMO-OFDM indoor visible light communications," IEEE Photon. Technol. Lett., vol. 28, no. 8, pp. 907-910, Apr. 2016.

[11] O. Narmanlioglu, R. C. Kizilirmak, T. Baykas, and M. Uysal, "Link adaptation for MIMO OFDM visible light communication systems," IEEE Access, vol. 5, pp. 26006-26014, Nov. 2017.

[12] Y. S. Cho, J. Kim, W. Y. Yang, and C. G. Kang, MIMO-OFDM Wireless Communications with MATLAB. Singapore: Wiley, 2010.

[13] E. Basar et al., "Index modulation techniques for next-generation wireless networks", IEEE Access, vol. 5, pp. 16693-16746, Sep. 2017.

[14] L. Bai and J. Choi, Low Complexity MIMO Detection. Springer, Boston, MA, USA, 2012

[15] M. T. Alresheedi, A. T. Hussein, and J. M. H. Elmirghani, "Uplink design in VLC systems with IR sources and beam steering," IET Commun., vol. 11, no. 3, pp. 311-317, Feb. 2017. 